Document downloaded from:

http://hdl.handle.net/10251/84397

This paper must be cited as:

Macho-Ortiz, A.; Morant Pérez, M.; Llorente Sáez, R. (2016). Next-Generation Optical Fronthaul Systems Using Multicore Fiber Media. Journal of Lightwave Technology. 34(20):4819-4827. doi:10.1109/JLT.2016.2573038.

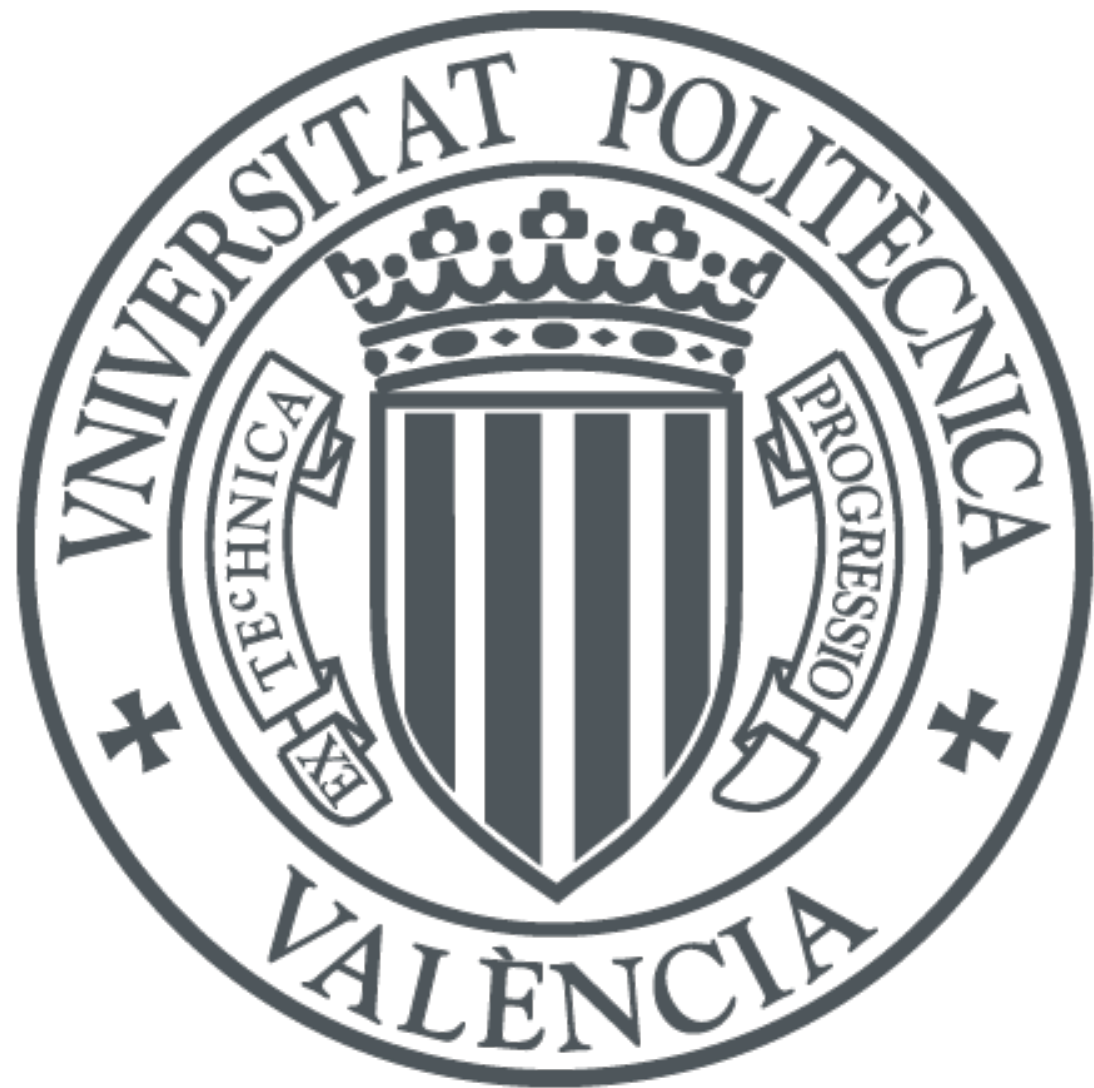

The final publication is available at

http://dx.doi.org/10.1109/JLT.2016.2573038

Copyright Institute of Electrical and Electronics Engineers (IEEE)

Additional Information

(c) 2016 IEEE. Personal use of this material is permitted. Permission from IEEE must be obtained for all other users, including reprinting/ republishing this material for advertising or promotional purposes, creating new collective works for resale or redistribution to servers or lists, or reuse of any copyrighted components of this work in other works. 


\title{
Next-generation Optical Fronthaul Systems using Multicore Fiber Media
}

\author{
Andrés Macho, Student Member, IEEE, Maria Morant, Member, IEEE, and \\ Roberto Llorente, Member, IEEE
}

\begin{abstract}
This paper proposes and investigates the use of multicore fiber (MCF) media performing space-division multiplexed transmission for next-generation optical fronthaul systems. We report the experimental demonstration of combined radio-over-fiber (RoF) transmission of full-standard LTE-Advanced (LTE-A) and WiMAX signals providing fronthaul connectivity in $150 \mathrm{~m}$ of 4-core fiber (4CF), transmitting simultaneously fully independent wireless services. Operating in linear and nonlinear optical power regimes, the experimental evaluation verifies that the error vector magnitude (EVM) is not degraded when inter-core and intra-core Kerr nonlinearities are excited in MCF with high input power levels. As a result, nonlinear regime is proposed as a key factor to reduce the temporal EVM fluctuation induced by the random nature of the inter-core crosstalk (IC-XT) in MCF. In addition, MCF fronthaul applied to converged fiber-wireless polarization multiplexed passive optical networks is demonstrated to transmit LTE-A and WiMAX signals over two orthogonal optical polarizations. The polarization-multiplexed signal is transmitted in RoF over $25.2 \mathrm{~km}$ of standard single-mode fiber and then demultiplexed and injected in different cores of the $4 \mathrm{CF}$ to provide fronthaul connectivity. Finally, the extension of multicore optical fronthaul capacity is proposed using MIMO LTE-A signals. The tolerance of the MIMO LTE-A RoF transmissions to in-band crosstalk is reported and compared to single-input singleoutput (SISO) configuration. The experimental results indicate that MIMO configuration is more tolerant than SISO to in-band crosstalk considering both internal and external interferences. MIMO and SISO configurations are compared when transmitted in RoF over a 4CF operating in linear and nonlinear regimes and core interleaving nonlinear stimulation (CINLS) is proposed to reduce the temporal and spectral EVM fluctuation when the same wireless standard is propagated in each core.
\end{abstract}

Index Terms - Multicore fiber, polarization multiplexing, multiple-input multiple-output (MIMO), radio-over-fiber

\section{INTRODUCTION}

$\mathrm{S}$ PACE-DIVISION MULTIPLEXING (SDM) systems using multicore fiber (MCF) media including different cores in a single cladding and additional peripheral devices for optical

Manuscript received January 15, 2016; revised May 1, 2016; accepted May 1, 2016. This research was supported in part by Spain the National Plan Project XCORE TEC2015-70858-C2-1-R and RTC-2014-2232-3 HIDRASENSE. The work of A. Macho was supported by BES-2013-062952 F.P.I. Grant. M. Morant was partly supported by UPV postdoc PAID-10-14 program.

A. Macho, M. Morant and R. Llorente are with Nanophotonics Technology Centre, Universitat Politècnica de València, 46022 Valencia, Spain, (e-mail: amachor@ntc.upv.es; mmorant@ntc.upv.es; rllorent@ntc.upv.es). connection have been extensively investigated in recent years targeting to overcome the exponential growth of data traffic in the backbone and in the access network [1]-[4]. Cloud radioaccess network (C-RAN) systems should also deal with this huge future capacity demand in the next-generation wireless systems, e.g. 5G cellular technology and Beyond-5G [5]-[7]. According some telecom equipment manufacturers, it is expected that $5 \mathrm{G}$ cellular networks will be required to provide 1000 times higher mobile data traffic in 2025 as compared with 2013, including flexibility and adaptability solutions to maximize the energy efficiency of the network [8],[9]. A new radio-access model supporting massive data uploading will be required considering additional transport facilities provided by the physical layer [8]-[10].

Fronthaul connectivity performed by radio-over-fiber (RoF) transmission using single-input single-output (SISO), multiple-input multiple-output (MIMO) configuration [11], sub-Nyquist sampling [12], and ultra-wideband signals exceeding $400 \mathrm{MHz}$ bandwidth has been proposed for the $5 \mathrm{G}$ cellular generation [6], [7], [13]. The required channel capacity is further extended in the case of Beyond-5G systems, where a massive number of antennas operating in MIMO configuration, should be connected using RoF. To overcome the massive increment in the data capacity demand, MCF has been recently proposed as a suitable medium for LTE-Advanced (LTE-A) MIMO fronthaul systems [13], [15].

MCF media opens up attractive possibilities in RoF systems as different wireless signals can be transmitted simultaneously over the same optical wavelengths and electrical frequencies in different cores of the optical waveguide to provide multi-wireless service using a single laser at the transmitter. Thus, MCF can also be proposed as an alternative to the standard single-mode fiber (SSMF) providing fronthaul connectivity using multiple wavelength channels with multiple lasers. Additionally, MCFs with high core density are suitable for connecting large phase array antennas performing multiuser MIMO (MU-MIMO) processing [16]. Furthermore, network operators can offer a dynamic and scalable capacity in the next cellular generation due to the aggregated channel capacity provided by the MCF technology [17]. Moreover, the possibility of combining MCF-RoF transmissions with additional multiplexing techniques as wavelength-division multiplexing (WDM), time-division multiplexing (TDM), optical polarization multiplexing (Pol-Mux) and mode-division multiplexing (MDM) [18] should be considered. Fig. 1 depicts the proposed fronthaul provision applied to converged fiber- 


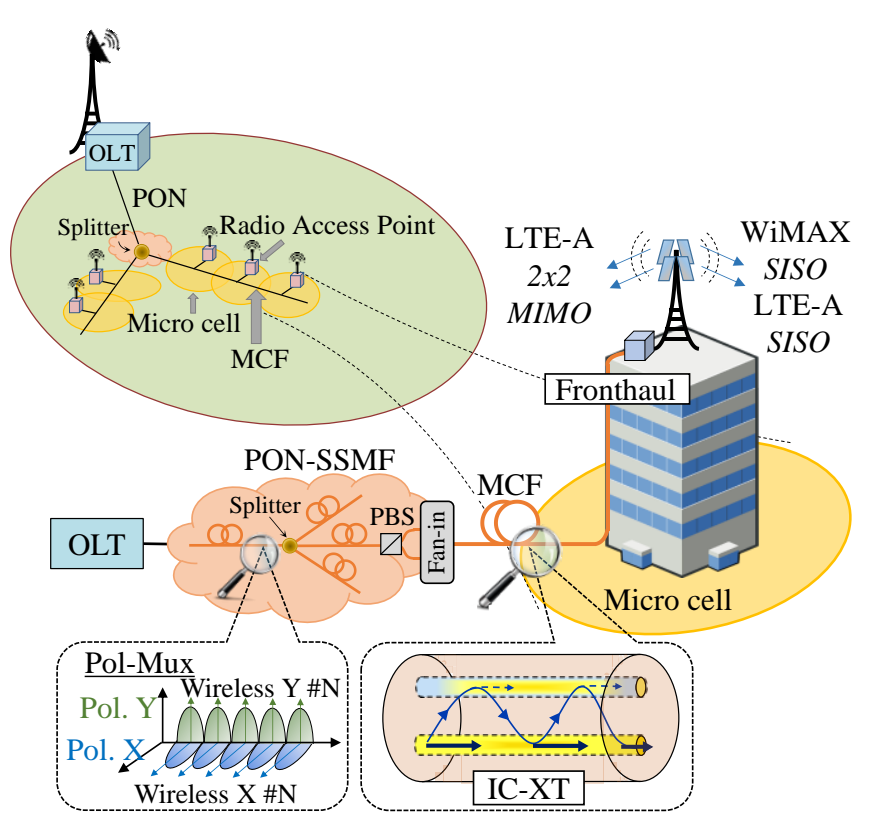

Fig. 1. Next-generation optical fronthaul system using multi-core fiber (MCF) media operating with a converged fiber-wireless PON including optical polarization-multiplexing transmission.

wireless passive optical networks (PON) including Pol-Mux to provide connectivity between the standard single-mode fiber (SSMF) and MCF media. Moreover, MCF transmission should also deal with the inter-core crosstalk (IC-XT) as the major physical impairment, which presents a stochastic nature due to fiber perturbations in linear and nonlinear regimes [19]-[21].

Targeting the use of MCF in the next-generation RoF fronthaul systems, this paper reports the experimental evaluation of the performance of fully-standard LTE-A signals in MIMO and SISO configurations with the IC-XT and the demonstration of fronthaul provision of both LTE-A and WiMAX signals using spatial multiplexing in a MCF. Due to the random nature of MCF perturbations, the error vector magnitude (EVM) also presents a stochastic behaviour. This paper further investigates the EVM random performance in these wireless standards and its impact in next-generation MCF-RoF systems.

The paper is structured as follows. In Section II, we demonstrate experimentally the provision of multi-wireless service fronthaul connectivity in MCF media using simultaneous RoF transmission of LTE-A and WiMAX signals. Considering the interoperability between both wireless standard [22], WiMAX is proposed as fallback option to provide connectivity in wireless scenarios where LTE-A service is overloaded [23]. In addition, the impact of IC-XT impairment over the RoF transmission is evaluated in a homogeneous 4-core fiber (4CF) when operating in linear and nonlinear optical power regimes. The EVM performance is evaluated in both power regimes to investigate the impact of inter-core and intra-core Kerr effects. The effective advantage of stimulating Kerr effect to reduce EVM randomization is proposed and demonstrated. In Section III, the application of
Pol-Mux of LTE-A and WiMAX signals as an extension capacity of the optical access network using additional MCF fronthaul provision is demonstrated. Next, in Section IV, the MCF RoF fronthaul transmission is evaluated using $2 \times 2$ MIMO built-in processing in LTE-A standard signals. In order to investigate the tolerance of SISO and MIMO configurations to the IC-XT impairment when transmitted in RoF over MCF, the EVM degradation due to in-band crosstalk of both configurations is analyzed and compared. Later, MCF evaluation of MIMO and SISO LTE-A RoF transmissions is performed operating in linear and nonlinear regimes. Finally, in Section VI, the main conclusions of this work are highlighted.

\section{Multi-WIRELESS LTE-A AND WiMAX Fronthaul RoF PROVISION USING MCF}

Most of the recent RoF research for next $5 \mathrm{G}$ networks is based on current wireless services such as 4G 3GPP LTE-A for evolved universal terrestrial radio access (E-UTRA) and IEEE 802.16 WiMAX orthogonal frequency division multiplexing access (OFDMA) for local and metropolitan area networks [10]. The simultaneous transmission of LTE-A signals with additional wireless standards permits delivering fixed, portable and mobile wireless services providing the new required multimedia services based on scalable video distribution or massive data uploading [7], [10]. In this scenario WiMAX service could also be used as LTE fallback option to provide reliable service even with overloading throughput. In order to demonstrate the compatibility of LTEA signals with additional wireless standards, we propose in this section the experimental demonstration of multiservice fronthaul provision using LTE-A and WiMAX signals in 150 $\mathrm{m}$ of a $4 \mathrm{CF}$ operating in linear and nonlinear power regimes.

Fig. 2 shows the experimental setup where full-standard wireless LTE-A and WiMAX signals are transmitted using the same optical carrier generated by a continuous wave (CW) distributed feedback laser (DFB) at $1550.12 \mathrm{~nm}$ and divided by a 3-dB optical coupler. Two external single-drive Mach-Zehnder modulators (MZMs) operating at quadrature bias (QB) point modulate the LTE-A and WiMAX signals in its native frequency and modulation format for RoF transmission. In order to stimulate linear and nonlinear regimes in MCF, the optical power level launched into each core is modified using two erbium-doped fiber amplifiers (EDFAs) with two band-pass optical filters (BPOFs) previously to the MCF signal injection. Four independent data signals, transmitted one in each core of the $4 \mathrm{CF}$, are generated employing two optical delay lines (ODLs). In addition, two variable optical attenuators (VOAs) are inserted to balance the optical power level launched into each core. The mode field diameter of the $4 \mathrm{CF}$ employed in the RoF transmissions (Fibercore SM-4C1500-8.0/125) is $8.4 \mu \mathrm{m}$, the numerical aperture is 0.15 , the cut-off wavelength is $1410 \mathrm{~nm}$ and it was spooled with an average diameter of $670 \mathrm{~mm}$ on a reel and a twist rate of 4 turns $/ \mathrm{m}$. The optical signals are injected and 


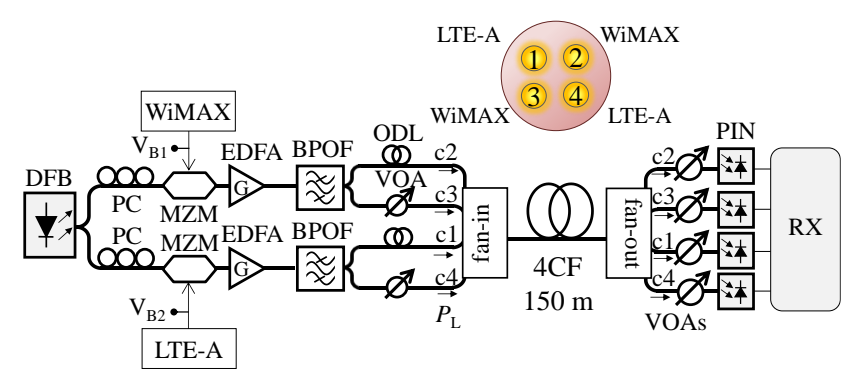

Fig. 2. Experimental set-up employed for the performance evaluation of LTE-A and WiMAX RoF transmission in 4CF.

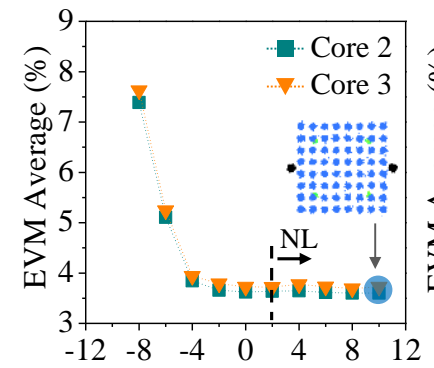

(a) Power Launch (dBm)

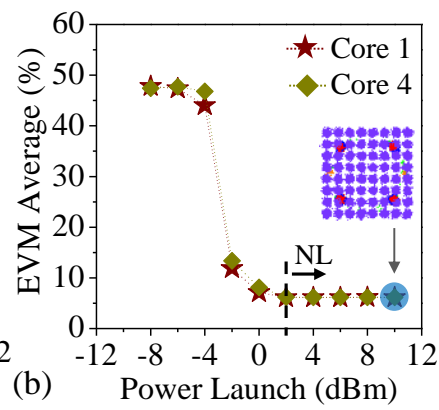

(b)
Fig. 3. Measured EVM average value vs optical power level launched into the cores of 4CF for: (a) WiMAX MCF-RoF transmission in cores 2 and 3, (b) LTE-A MCF-RoF transmission in cores 1 and 4. NL: nonlinear regime.
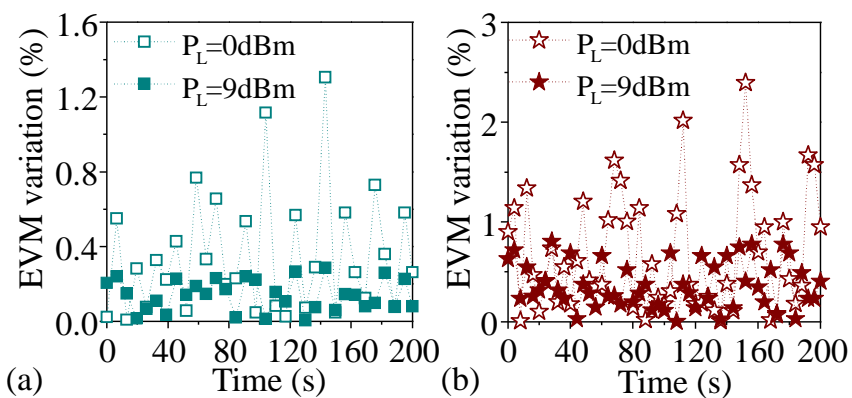

Fig. 4. Time EVM fluctuation measured for: (a) WiMAX in core 3 and (b) LTE-A in core 1, for MCF-RoF operating in linear regime (hollow symbols) and in nonlinear regime (solid symbols).

extracted from each core with a 3D fan-in/fan-out with an insertion loss of $4.5 \mathrm{~dB}$. After the MCF-RoF transmission, four independent PIN photodiodes with $0.8 \mathrm{~A} / \mathrm{W}$ responsivity are used to perform direct detection of the received signals, which are sampled with a real-time oscilloscope DSO91304A and demodulated with Agilent 89600B VSA software.

The LTE-A and WiMAX signals are generated by Agilent ESG 4438C generators following each wireless standard. The 3GPP LTE-A signal comprises two frequency division duplex (FDD) channels of $20 \mathrm{MHz}$ each one with 100 resource blocks (RBs) centered at 2.633 and $2.655 \mathrm{GHz}$ frequencies in the 3 GPP band 7, respectively. In addition, the WiMAX signal comprises three IEEE 802.16 FDD channels of $20 \mathrm{MHz}$ bandwidth centered at $3.478,3.5$ and $3.522 \mathrm{GHz}$, respectively. The LTE-A signals are configured with normal cyclic prefix and WiMAX signals with $1 / 8$ cyclic prefix. Four LTE-A frames of $10 \mathrm{~ms}$ each and five WiMAX frames of $5 \mathrm{~ms}$ are generated using 64 quadrature amplitude modulation
(64QAM) in each OFDM subcarrier.

The EVM performance of WiMAX and LTE-A MCF-RoF transmission in linear and nonlinear power regimes is depicted in Fig. 3 including some examples of the received 64QAM constellations. The EVM average values were measured as a function of the optical power launch level. It can be noticed that the optimal EVM average performance is achieved with a power launch level higher than $0 \mathrm{dBm}$ for WiMAX signals and $2 \mathrm{dBm}$ for LTE-A signals. In addition, we verified that the EVM performance of LTE-A and WiMAX channels is not degraded when operating in nonlinear regime with optical power launch levels higher than $2 \mathrm{dBm}$, which was found as the critical power of silica MCFs as detailed in [20]. Moreover, it should be remarked that the IC-XT average value increases as the bending radius increases [15]. However, it is expected a similar EVM performance when using higher bending radius than $670 \mathrm{~mm}$ in the $4 \mathrm{CF}$ considering that the maximum IC-XT average value was estimated lower than $-40 \mathrm{~dB}$ when using a bending radius of $1000 \mathrm{~mm}$ [21].

As we verify in Fig. 4, the stimulation of nonlinear regime is an attractive strategy in MCF-RoF systems to reduce the EVM time fluctuation due to IC-XT impairment. IC-XT presents a random nature in both linear and nonlinear power regimes due to the random perturbations of MCF [19]-[21]. Therefore, we can also observe stochastic fluctuations in the EVM performance induced by the instantaneous fluctuations of the optical power exchanged among cores. However, when stimulating a single core with a high input power level, Kerr effect mismatches the phase constant of adjacent core modes reducing the IC-XT mean and variance [20]. Consequently, the instantaneous EVM fluctuation is reduced in LTE-A and WiMAX signals when operating with high optical power launch levels in a single core of each wireless standard, as it is experimentally confirmed in Fig. 4.

Figs. 4(a) and (b) show the temporal EVM fluctuation percentage (calculated respect to the EVM average value) measured in cores 1 and 3 for WiMAX and LTE-A signals, respectively, when injecting an optical signal of $0 \mathrm{dBm}$ and $9 \mathrm{dBm}$ in both cores. As a result, the phase constants of $\mathrm{LP}_{01}$ mode between cores 1-4 and 2-3 are mismatched reducing the power exchange between cores carrying similar wireless standard. The experimental results indicate that EVM time fluctuation is reduced in WiMAX and LTE-A MCF-RoF transmissions when mismatching cores 1-4 and 2-3 stimulating fiber Kerr nonlinearities.

Moreover, an additional consideration should be pointed out in the fronthaul system proposed in this section. As can be noticed from Fig. 2, a single DFB laser was employed to minimize the energy consumption in the RoF transmission. However, an alternative approach can also be proposed using four independent DFBs with four different optical carriers providing more tolerance to hardware failures. In addition, the in-band crosstalk induced by the IC-XT becomes out-band crosstalk reducing the EVM performance degradation in more critical scenarios as in coupled MCFs. 


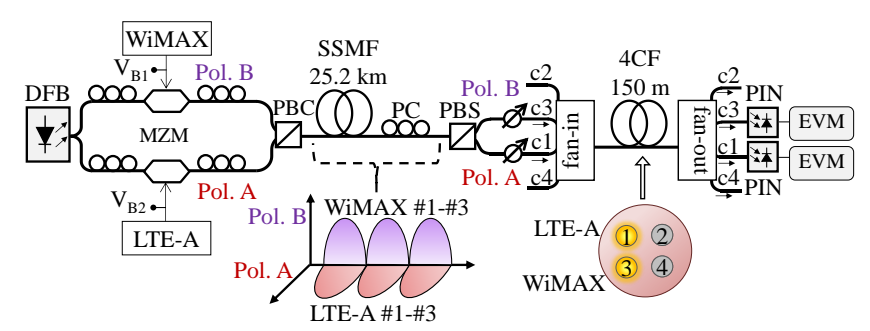

Fig. 5. Laboratory set-up for the experimental demonstration of PON extension capacity by using polarization-multiplexing (Pol-Mux) of LTE-A and WiMAX signals in $25.2 \mathrm{~km}$ SSMF and optical fronthaul provision in $150 \mathrm{~m}$ of 4 -core fiber $(4 \mathrm{CF})$.

\section{POL-MUX PON EXTENSION CAPACITY USING ROF AND MCF FRONTHAUL PROVISION}

Converged fiber-wireless access using RoF in PON have been proposed and developed in the last decade to connect the base station (BS) to the remote radio head (RRH) [24]. As expected in next $5 \mathrm{G}$ networks, a large number of RRHs providing high wireless capacity will be required. Therefore, converged fiber-wireless PON systems using RoF transmissions should also be developed to meet this huge capacity demand [5]. In order to confirm the suitability of the MCF fronthaul system proposed in the previous section applied to converged fiber-wireless PON systems using SSMF, in this section we evaluate experimentally the transmission of LTE-A and WiMAX signals over a Pol-Mux PON followed by MCF-RoF optical fronthaul. The laboratory set-up employed to evaluate the Pol-Mux PON and the MCF-RoF fronthaul is depicted in Fig. 5. The proposed RoF system maximizes the PON capacity by aggregating three wireless carriers over two orthogonal optical polarizations followed by MCF-RoF fronthaul connectivity. Three $20-\mathrm{MHz}$ LTE-A carriers are transmitted using polarization A (Pol. A) and three $20-\mathrm{MHz}$ WiMAX carriers are transmitted using orthogonal polarization (Pol. B) of the same wavelength centred at $1555.75 \mathrm{~nm}$.

The RoF performance is evaluated under the worst-case in-band interference scenario by selecting the same radiofrequency band centred at $2.5 \mathrm{GHz}$ in both wireless standards, as commercially available in $4 \mathrm{G}$ multi-wireless service devices [10]. The $1555.75 \mathrm{~nm}$ optical carrier is generated by a CW DFB and divided by a 3-dB optical splitter in two paths. RoF transmission is achieved by modulating the full-standard LTE-A and WiMAX signals with MZMs at QB point in the upper and the lower path, respectively. A polarization beam combiner (PBC) is employed to inject both polarizations in a $25.2 \mathrm{~km}$ of SSMF.

After PON transmission both polarizations are demultiplexed with a polarization beam splitter (PBS) and each wireless standard is transmitted over a different core of the same MCF employed in Section II. Adjacent cores 1 and 3 were employed to maximize the IC-XT average level around $-43 \mathrm{~dB}$ [20]. The optical power level launched into each core from the PON was varied from -13 to $-6 \mathrm{dBm}$ using two VOAs. At the optical receiver, direct detection was performed

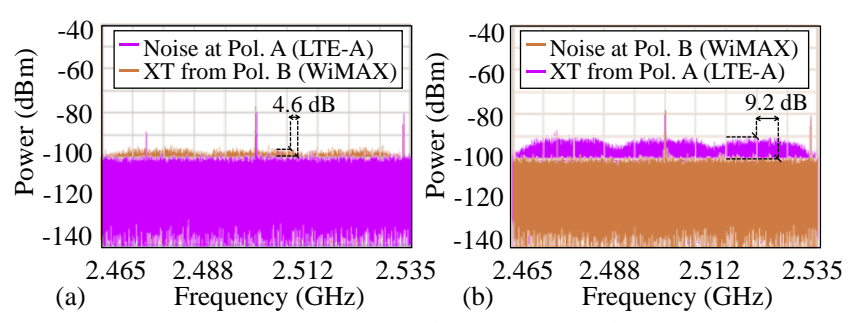

Fig. 6. Measured electrical spectra of cross-polarization crosstalk versus noise floor after $25.2 \mathrm{~km} \mathrm{SSMF} \mathrm{PON} \mathrm{and} 150 \mathrm{~m} \mathrm{4CF}$ transmission for: (a) Pol. A carrying three 20-MHz LTE-A carriers and (b) Pol. B with three $20-\mathrm{MHz}$ WiMAX carriers.

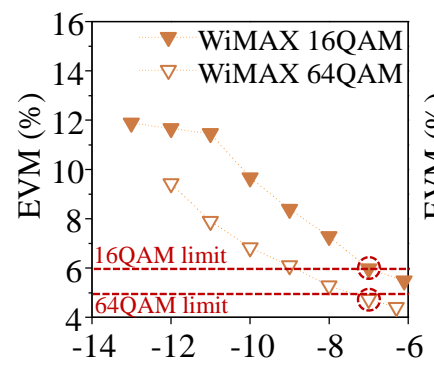

(a) Optical Power (dBm)

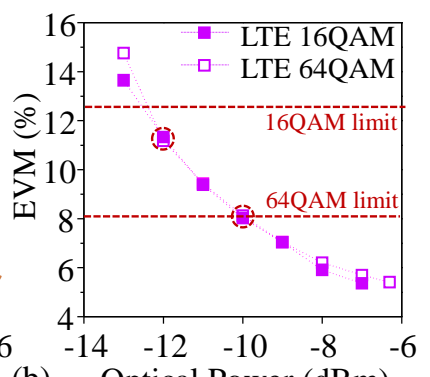

Fig. 7. Measured EVM at the receiver vs. optical power level launched into cores 1 and 3 for: (a) WiMAX and (b) LTE-A RoF transmission.

using two PIN photodetectors and each RF signal was sampled and demodulated as in Section II. The measured EVM was compared with the wireless standard recommendations at the transmitter antenna for each subcarrier modulation order: $\mathrm{EVM}_{16 \mathrm{QAM}}<12.5 \%$ and $\mathrm{EVM}_{64 \mathrm{QAM}}<8 \%$ for 3GPP LTE-A, and $\mathrm{EVM}_{16 \mathrm{QAM}}<6 \%$ and $\mathrm{EVM}_{64 \mathrm{QAM}}<5 \%$ for IEEE 802.16 WiMAX.

The measured spectra at both cores with/without the presence of the orthogonal optical polarization in the PON network is shown in Fig. 6. Fig. 6(a) indicates that a maximum cross-polarization crosstalk of $4.6 \mathrm{~dB}$ is detected in core 1 (LTE-A transmission in Pol. A) due to interference from Pol. B (WiMAX transmission) generated in the SSMF and in the PBS due to a potentially imperfect operation. The crosstalk measured in core 3 for WiMAX receiver (Pol. B) is $9.2 \mathrm{~dB}$ from the interference coming from Pol. A also generated in the SSMF and PBS, as depicted in Fig. 6(b).

Fig. 7 shows the measured EVM for both LTE-A and WiMAX signals after the MCF-RoF fronthaul provision using $150 \mathrm{~m}$ of $4 \mathrm{CF}$ as a function of the optical power level launched into each core. The successful transmission should be noticed for the three LTE-A and WiMAX channels carried by each polarization and each core in the SSMF and 4CF. 3GPP LTEA antenna limit is satisfied for the three LTE-A channels carried by Pol. A and core 1 when operating with an optical power level launched into core 1 higher than $-9 \mathrm{dBm}$ for 64QAM carrier modulation. The required optical power launch is reduced to $-11 \mathrm{dBm}$ when using 16QAM subcarrier modulation over the same bandwidth in the carrier-aggregated RoF transmission. Moreover, considering the WiMAX RoF transmission carried by Pol. B and core 3, the optical power launch required in core 3 to meet the IEEE 802.16 WiMAX 
antenna limit is increased to $-7 \mathrm{dBm}$ for both 16QAM and 64QAM subcarrier modulations. It should be noticed that WiMAX requires higher optical power operation levels due to the higher cross-polarization interference observed in Pol. B.

\section{FRONTHAUL EXTENSION CAPACITY IN MCF-ROF: IMPROVEMENT USING MIMO PROCESSING}

As we commented before, MCF fronthaul capacity can be extended using RoF transmission and MIMO processing. However, in MCF the random power exchange among cores due to IC-XT and its impact in the additional signal processing of MIMO MCF-RoF transmissions should be investigated and compared with SISO configuration. The $2 \times 2$ MIMO processing algorithm used in this experimental evaluation is implemented in current LTE-A devices meeting 3GPP standard for wireless multipath compensation.

Employing different wavelengths in each excited core of the MCF, the IC-XT generates out-band crosstalk minimizing the EVM degradation due to mode coupling among cores. However, in order to reduce the energy consumption in the RoF transmission, a single optical carrier is employed to illuminate the cores of the MCF and, therefore, the IC-XT becomes in-band crosstalk. In this section, we first analyze the tolerance of LTE-A RoF transmissions to in-band crosstalk comparing both MIMO and SISO configurations, and later, the performance of MIMO and SISO LTE-A is investigated in MCF media using a single optical carrier to illuminate the different cores of the fiber.

\section{A. Tolerance of LTE-A RoF Transmissions to In-Band Crosstalk}

In-band crosstalk is referred as the interference between two different signals using the same central frequency. In the same way, IC-XT can be classified as in-band crosstalk when using the same optical carrier in each core. The experimental evaluation of the LTE-A RoF tolerance to in-band crosstalk becomes neccesary to understand the propagation performance of LTE-A RoF transmissions in MCF media.

If we consider a 2x2 MIMO LTE-A transmission using spatial multiplexing, we refer to crosstalk induced by internal interference to the crosstalk between the master and slave signals containing different data that compose the $2 \times 2$ MIMO LTE-A [25]. In contrast, the crosstalk induced by external interference refers to the crosstalk between different signals, e.g. the $2 \times 2$ MIMO signal interfering with other external source. In this case, in order to evaluate the worst-case scenario, we use different signals with the same central frequency and bandwidth to have in-band interference. Consequently, in RoF transmissions using MCF media, the IC-XT of a $2 \times 2$ MIMO transmitter is referred as internal IC-XT, while the external IC-XT occurs among cores carrying signals from different transmitters.

The evaluation of the impact of in-band crosstalk in LTE-A RoF transmissions should be performed considering different crosstalk levels. In MCF media, IC-XT presents a random nature with low crosstalk variance [20], [21]. Therefore, the in-band crosstalk evaluation in LTE-A RoF transmissions

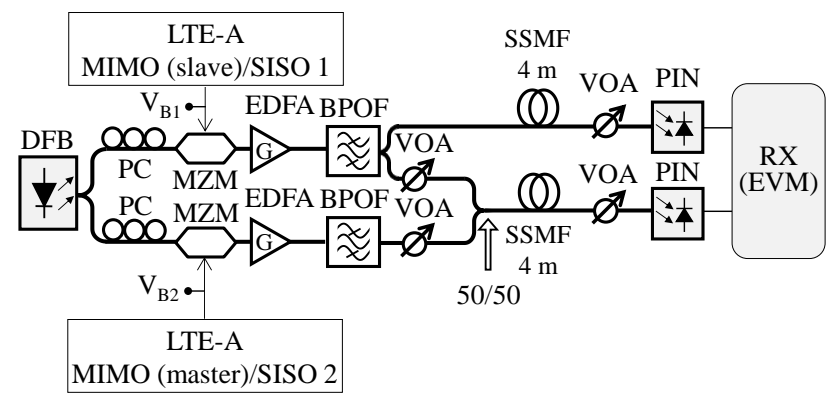

Fig. 8. Experimental set-up employed for the evaluation of the in-band crosstalk induced by internal interference in RoF LTE-A MIMO and SISO transmissions.

should be performed using optical couplers and standard single-mode fiber (SSMF) in order to force higher crosstalk levels than in a MCF with constant core pitch.

The LTE-A signals evaluated in this experiment are generated as in the previous sections, with a $2.655 \mathrm{GHz}$ central frequency using the maximum LTE channel bandwidth of $20 \mathrm{MHz}$. In this case we compare the RoF performance using SISO and MIMO transmission. LTE-A 2x2 MIMO transmission is configured with spatial multiplexing considering 2 layers without cyclic delay diversity.

Considering both in-band crosstalk cases (internal and external interference), it should be remarked that the experimental evaluation is performed with the same LTE-A SISO signals using the same modulation order and bandwidth as the MIMO configuration. The comparison of the crosstalk tolerance of MIMO and SISO RoF transmission should be additionally performed to evaluate the capability of the 3GPP MIMO algorithms to deal with the in-band crosstalk impairment when using MCF-RoF. Furthermore, it should be noticed that using $2 \times 2$ MIMO configuration, two layers of different data are spatial multiplexed compared with SISO transmission. Therefore, almost double bitrate is provided in MIMO compared with SISO configuration over the same bandwidth and with the same modulation order.

The tolerance of 2x2 MIMO LTE-A RoF transmissions to internal interference between the master and slave signals is evaluated using the experimental set-up depicted in Fig. 8. The optical system emulates a $2 \times 2$ MIMO transmission using a single optical carrier at $1550.12 \mathrm{~nm}$ and divided in two paths with a 3-dB optical splitter. Each path is modulated by the LTE-A signal using a single-drive MZM operating at QB point. Additional EDFAs, BPOFs and VOAs are included in the laboratory set-up to induce high crosstalk levels in the optical signals. Two short standard single-mode fiber (SSMF) patch cords with 4-m length were employed to emulate a MCF propagation scenario with tunable IC-XT average value. The internal interference is extracted from the upper path and injected into the lower path with a 3-dB optical coupler. The VOAs at the transmitter are used to control the in-band crosstalk level induced in the lower path and the VOAs at the receiver are used to keep balanced the received optical power in each photodiode.

The internal interference in MIMO and the external 


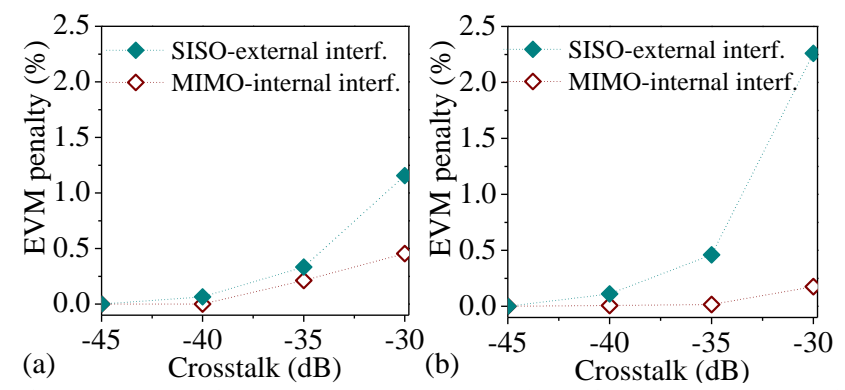

Fig. 9. Measured RoF LTE-A EVM penalty using: (a) 16QAM and (b) 64QAM subcarrier modulation.

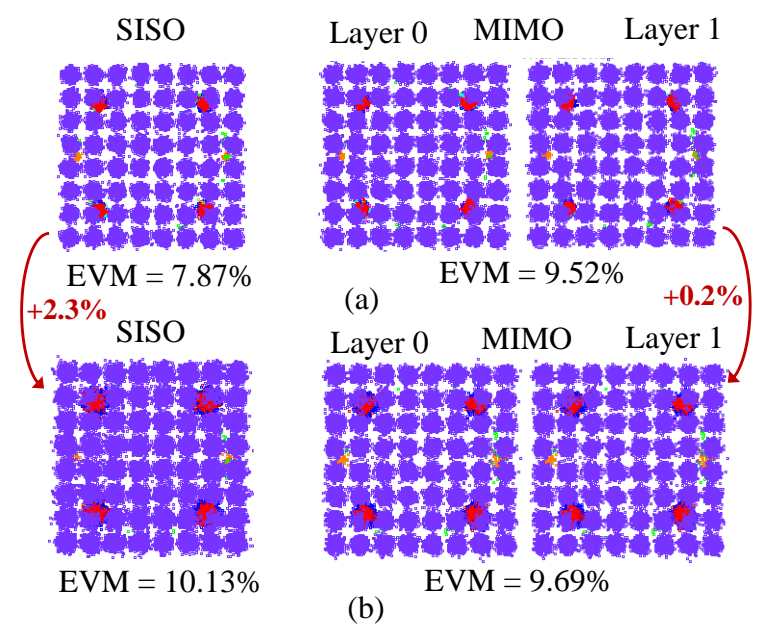

Fig. 10. Measured constellations of SISO and 2x2 MIMO 64QAM LTE-A with: (a) $\mathrm{EVM}_{\text {penalty }}=+2.3 \%$ and (b) $\mathrm{EVM}_{\text {penalty }}=+0.2 \%$.

interference in SISO are evaluated modifying the crosstalk level from -45 to $-30 \mathrm{~dB}$ in the lower path. The experimental measurements depicted in Figs. 8 and 11 correspond to the lower optical path of each laboratory setup. The crosstalk tolerance of both scenarios is analyzed calculating the EVM penalty as a function of the induced crosstalk level. The EVM penalty is calculated as the difference between the EVM (in \%) obtained with the minimum crosstalk level $(\mathrm{XT}=-\infty)$ and the EVM (in \%) measured with the current crosstalk level induced with the VOAs and the optical coupler.

Figs. 9(a) and (b) show the measured EVM penalty as a function of the internal crosstalk level when using 16QAM and 64QAM subcarrier modulations, respectively. It can be observed that the EVM penalty in MIMO presents a lower slope than in SISO. Therefore, the EVM performance of SISO configuration presents a higher degradation than MIMO for each in-band crosstalk level. As a result, we conclude that MIMO transmission presents a better tolerance than SISO configuration to in-band crosstalk. These results confirm the successful tolerance of MIMO LTE-A transmission to in-band crosstalk induced by internal interference. In addition, Fig. 10 shows the impact in the received 64QAM constellations of -30 $\mathrm{dB}$ of in-band crosstalk generated by internal interference in MIMO and external interference in SISO. The digital constellations confirm that $2 \times 2$ MIMO LTE-A shows better tolerance than SISO when the in-band crosstalk level is increased. Consequently, it is expected that LTE-A RoF transmission in coupled MCF using MIMO will present a

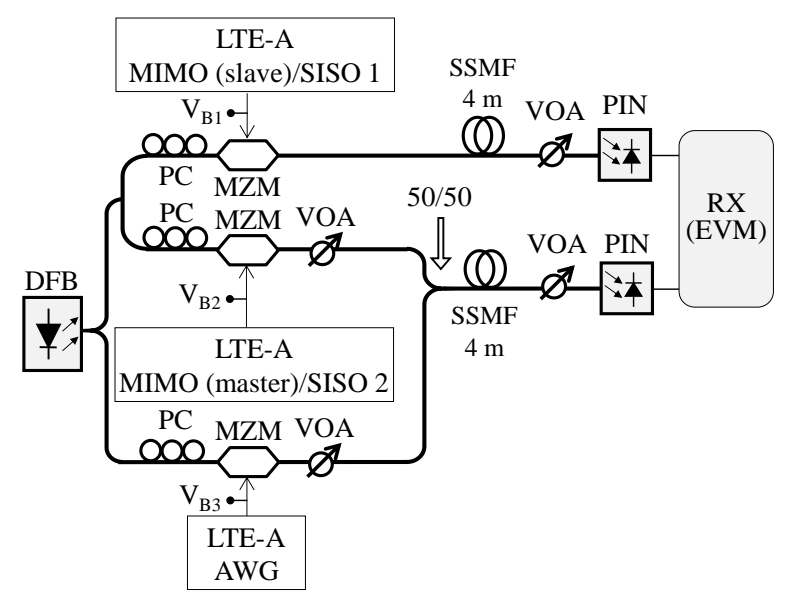

Fig. 11. Experimental set-up used for the evaluation of in-band crosstalk induced by external interference in RoF LTE-A MIMO and SISO transmissions.
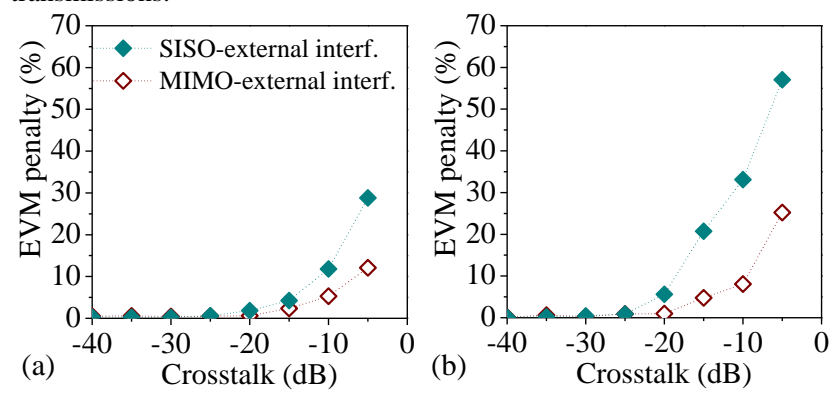

Fig. 12. Measured RoF LTE-A EVM penalty using: (a) 16QAM and (b) 64QAM subcarrier modulation.

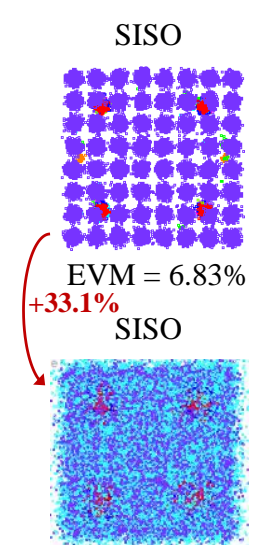

$\mathrm{EVM}=39.92 \%$

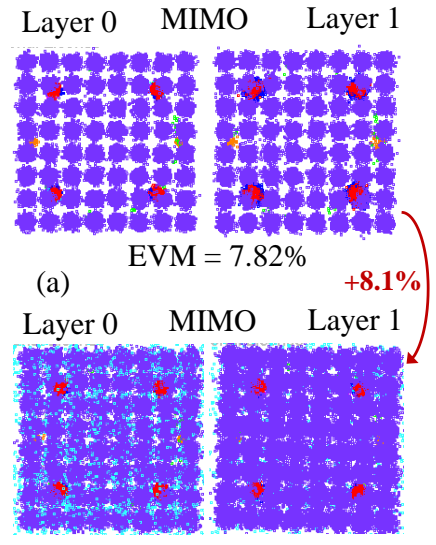

(b)
Fig. 13. Measured constellations of SISO and 2x2 MIMO 64QAM LTE-A with: (a) $\mathrm{EVM}_{\text {penalty }}=+33.1 \%$ and (b) $\mathrm{EVM}_{\text {penalty }}=+8.1 \%$.

better performance than SISO over the same bandwidth.

On the other hand, the tolerance analysis of the MIMO and SISO LTE-A signals to in-band crosstalk induced by an external interference is performed using the experimental set-up depicted in Fig. 11. Please note that the difference between Fig. 8 and Fig. 11 is based on the use of an external interfering signal in the latter case. The external interference is generated by an arbitrary waveguide generator (AWG) with the same electrical bandwidth $(20 \mathrm{MHz})$ and in the same frequency band (at $2.655 \mathrm{GHz}$ center frequency). In addition, it should be remarked that no EDFAs were included in this setup because it is not necessary to preserve the optical power 
budget between the two paths considering that the power level injected into the lower path is not extracted from the upper path in this case.

Figs. 12(a) and (b) show the EVM penalty as a function of the crosstalk level induced by external interference considering 16QAM and 64QAM subcarrier modulations, respectively. In both cases, MIMO presents a lower EVM penalty than SISO for any in-band crosstalk level induced by the external interfering signal. It should be noticed that the EVM penalty evolution presents a lower slope in MIMO than in SISO. Hence, the EVM performance presents a lower degradation in MIMO for each crosstalk value induced by the external interference. Consequently, MIMO configuration can be an interesting option to perform SDM optical transmissions in MCF media with high IC-XT levels when using high-order modulation formats. Furthermore, Fig. 13 depicts the degradation of digital constellations of the received 64QAM LTE-A signals in SISO and MIMO configurations when the power of the external interfering signal is increased. It can also be observed that SISO constellation is more degraded than MIMO, as was pointed out in Fig. 12(b).

\section{B. MCF-RoF fronthaul evaluation}

MCF fronthaul capacity can additionally be extended using RoF transmission of full-standard LTE-A signals in MIMO configuration. However, the power exchange among the excited cores and its impact in the additional signal processing of MIMO transmissions should be investigated and compared with SISO configuration. In this section, MIMO and SISO configurations of LTE-A signals are experimentally evaluated and compared in a homogeneous 4CF. The impact of linear and nonlinear IC-XT in the EVM performance is investigated reporting the effective advantage of stimulating Kerr effect in both MIMO and SISO configurations.

The experimental setup employed in this section using LTE-A signals is shown in Fig. 14. A single carrier is generated at $1550.12 \mathrm{~nm}$ and divided in two branches for optical external modulation. The optimum modulation index of the signal was selected in each case as performed in [13]. Two EDFAs and BPOFs are included previously to the MCF signal injection to stimulate linear and nonlinear regimes in the $4 \mathrm{CF}$, the same as in the previous sections spooled on a reel with a bending radius of $670 \mathrm{~mm}$.

Two different MCF-RoF configurations have been used to evaluate EVM fluctuation of LTE-A signals in linear and nonlinear regime: (\#1) comparison of MCF-RoF performance using SISO and $2 \times 2$ MIMO configurations for the evaluation of the EVM fluctuation when varying the optical power launched into the cores 1 and 3. And (\#2) MFC-RoF transmission of four independent SISO LTE-A signals for the evaluation of EVM fluctuation in the spectral domain using the four cores of the 4CF. Optical decorrelation of the four SISO LTE-A signals is performed using two ODLs and two VOAs to balance the optical power injected in each core. In both MCF-RoF configurations \#1 and \#2 the full-standard LTE-A wireless signals are centred at 2.633 and $2.655 \mathrm{GHz}$

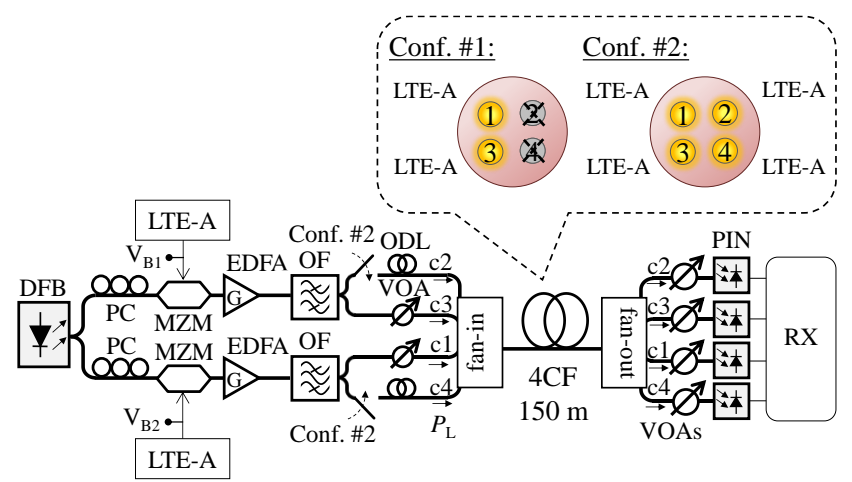

Fig. 14. Experimental set-up for LTE-A 2x2 MIMO and SISO MCF-RoF transmission using a homogeneous 4CF. Two different MCF-RoF configurations are employed: (\#1) using only cores 1 and 3, and (\#2) using the four cores of the $4 \mathrm{CF}$.
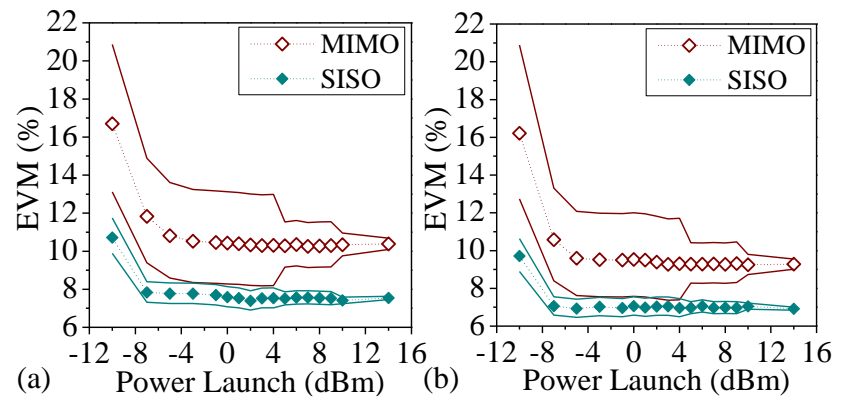

Fig. 15. Measured EVM average value (dots) and EVM fluctuation margins (solid lines) for $2 \times 2$ MIMO and SISO LTE-A MCF-RoF configuration \#1 considering: (a) 16QAM and (b) 64QAM subcarrier modulations.

frequencies in the $3 \mathrm{GPP}$ band 7.

Experimental measurements of MCF-RoF configuration \#1 for SISO and MIMO transmissions are depicted in Fig. 15. The LTE-A EVM performance was measured as a function of the optical power level launched into each core. The MIMO and SISO signals are transmitted in adjacent cores 1 and 3 to evaluate the RoF performance considering the highest IC-XT conditions. The EVM average value (dots) and the maximum and minimum EVM excursion (solid lines) were measured for different optical power levels launched into the cores 1 and 3. In linear regime, the optical power was increased from $-10 \mathrm{dBm}$ to $+2 \mathrm{dBm}$ in both cores. In nonlinear regime, in order to mismatch the phase constant of core modes, the optical power launch was maintained fixed in $+2 \mathrm{dBm}$ for core 3 and was increased from +2 to $+14 \mathrm{dBm}$ in core 1 .

It can be noticed from in Fig. 15 that MIMO configuration presents a higher EVM fluctuation than SISO and worse EVM average performance. In MCF media with uncoupled cores and low IC-XT average level, a better EVM performance can be achieved with SISO configuration for the same modulation order, but MIMO achieves higher data rate. Furthermore, stimulating a single core with high power levels, the index mismatching between cores 1 and 3 increases, which reduces the EVM fluctuation in both SISO and MIMO MCF-RoF configurations. This is confirmed in the EVM fluctuation margins depicted in Fig. 15. The higher the power level injected into core 1 (keeping constant the power level 


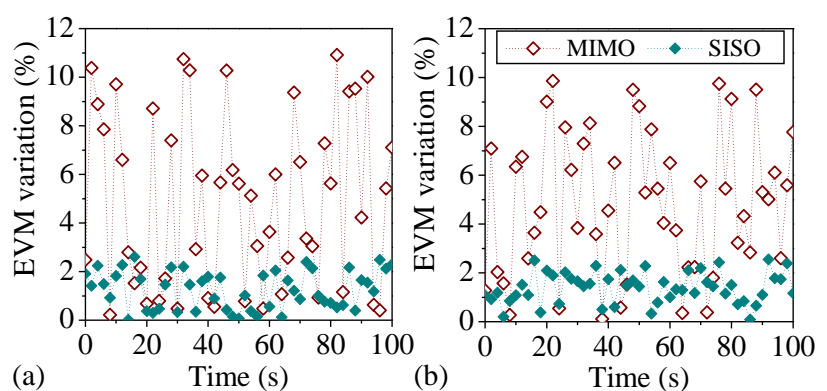

Fig. 16. Time EVM fluctuation for $2 \times 2$ MIMO and SISO LTE-A MCF-RoF configuration \#1 considering: (a) 16QAM and (b) 64QAM subcarrier modulations.
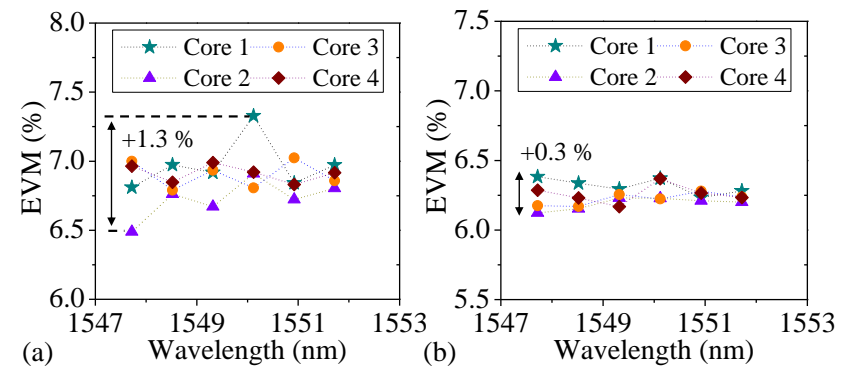

Fig. 17. Spectral EVM fluctuation measured in SISO LTE-A MCF-RoF configuration \#2 injecting an optical power launch level in cores 1 and 4 of: (a) $0 \mathrm{dBm}$ and (b) $9 \mathrm{dBm}$, with a fixed power launch level of $0 \mathrm{dBm}$ in cores 2 and 3 .

launched into core 3), the lower the EVM fluctuation in MIMO and SISO configurations due to the variance reduction of IC-XT in nonlinear regime. Additionally, as detailed in [20], the IC-XT mean is also reduced but the EVM average value is not improved due to the absence of crosstalk penalty in linear regime, as it was detailed in Section III.A. In addition, it should be remarked that if we operate with data bit rates below $1 \mathrm{~Gb} / \mathrm{s}$ the EVM performance is not degraded when intra- and inter-core Kerr nonlinearities are stimulated. Therefore, aimed to reduce the mean and variance of IC-XT, fiber Kerr nonlinearities can be stimulated in MCF-RoF transmissions.

Moreover, the EVM fluctuation difference between MIMO and SISO was additionally investigated in time domain operating in linear regime, where the EVM fluctuation difference is maximized, as depicted in Fig. 15. Fig. 16 shows the EVM percentage variation calculated respect to the EVM average level for 100 seconds considering $+0 \mathrm{dBm}$ of power launch per core. It is verified from the time analysis that the EVM fluctuation is higher in MIMO than in SISO. MIMO presents a maximum EVM excursion of $11 \%$, while the maximum excursion in SISO is around 3\%. Considering that the refreshing period of the MIMO channel matrix is every 42 LTE symbols in our experiments $(\sim 2.8 \mathrm{~ms})$, and the coherence time of the MCF structural fluctuations is lower than $1 \mathrm{~ms}$ [20], [21], MIMO presents higher EVM temporal fluctuation than SISO.

Finally, in MCF-RoF configuration \#2, the EVM fluctuation was evaluated in the wavelength domain. Due to the randomization of the phase constant of core modes when the wavelength of the optical carrier is modified, the EVM RoF performance is also randomized in the optical spectrum. It can be observed in Fig. 17(a) that the EVM performance varies significantly up to $+1.3 \%$ with the wavelength in linear regime. However, when stimulating nonlinear regime in cores 1 and 4 with a power launch level of $+9 \mathrm{dBm}$ operating simultaneously in cores 2 and 3 in linear regime injecting $+0 \mathrm{dBm}$, the phase constants of $\mathrm{LP}_{01}$ mode between adjacent cores are mismatched. This is confirmed with a reduction in the EVM performance difference between optical carriers from $+1.3 \%$ to $+0.3 \%$, as shown in Fig. 17(b). In both power regimes, all cores present an EVM below the EVM 3GPP limit $\left(\mathrm{EVM}_{16 \mathrm{QAM}}<12.5 \%\right)$, meeting the standard recommendation.

In long-reach WDM systems, the EVM fluctuation in wavelength domain should be considered as some optical channels could satisfy the requirements while others could be deeply affected. In this situation, the stimulation of adjacent cores carrying the same wireless standard with different optical power launch levels, also known as core interleaving nonlinear stimulation (CINLS), is appointed as an interesting strategy to avoid this limitation. CINLS induces a phase-mismatching between adjacent cores of the MCF using different optical power launch levels in each core. Hence, CINLS proposes to use the nonlinear Kerr effect to reduce the mean and variance of the IC-XT random process minimizing the temporal and spectral EVM fluctuation between adjacent cores of the MCF.

\section{CONCLUSION}

This paper investigates the use of MCF media in the next generation of optical fronthaul systems applied to 5G-cellular networks. We demonstrated the multi-wireless service fronthaul provision using LTE-A and WiMAX signals in MCF to provide reliable services connectivity. The experimental investigation of the MCF-RoF performance confirms that the optimal EVM average performance is achieved with a power launch level higher than $+0 \mathrm{dBm}$ for WiMAX signals and $+2 \mathrm{dBm}$ for LTE-A signals over $150 \mathrm{~m}$ of $4 \mathrm{CF}$. The EVM performance of LTE-A and WiMAX MCF-RoF transmissions is not degraded when operating with high optical power launch levels stimulating fiber Kerr nonlinearities. The experimental results confirmed that the nonlinear regime can be employed using a power launch level higher than the critical power of the MCF (+2 dBm for silica cores) to reduce the temporal EVM fluctuation observed between cores carrying the same wireless standard. Adjacent cores 1 and 3 were stimulated with $+9 \mathrm{dBm}$ reducing the EVM fluctuations between core 1-4 carrying LTE-A signals and core 2-3 carrying WiMAX signals. Furthermore, we proposed the suitability of the MCF fronthaul systems applied to converged fiber-wireless Pol-Mux PONs. LTE-A and WiMAX signals were transmitted over a Pol-Mux PON using a $25.2 \mathrm{~km}$ SSMF previous to the MCF fronthaul system.

Considering that the average value of the IC-XT in MCF is lower than the cross-polarization crosstalk in SSMF, MCF media is proposed to provide fronthaul connectivity as an 
evolution of the conventional RoF systems. The suitability of in-built 3GPP LTE-A MIMO processing for MCF-RoF transmission was also evaluated experimentally. The tolerance of the LTE-A RoF transmissions to in-band crosstalk was evaluated considering both internal and external interferences in both SISO and $2 \times 2$ MIMO LTE-A RoF transmissions. It was observed that MIMO processing supports high-levels of in-band crosstalk induced by internal and external interference. Therefore, MIMO processing provides better tolerance than SISO to IC-XT. Finally, SISO and MIMO LTE-A MCF-RoF transmissions were compared when operating in linear and nonlinear optical regimes. Although MIMO presents better tolerance to IC-XT than SISO, lower EVM fluctuations induced by MCF random perturbations were observed in SISO LTE-A RoF transmission considering that the coherence time of the MCF structural fluctuations is lower than the refreshing period of the MIMO channel matrix. Finally, CINLS strategy was proposed to mismatch the phase constant of adjacent core modes reducing the temporal and spectral EVM fluctuations of SISO and 2x2 MIMO LTE-A MCF-RoF transmissions.

The low crosstalk observed between adjacent cores of the $4 \mathrm{CF}$ indicates that the major physical impairment in the RoF transmissions is induced by the power insertion losses of the 3D fan-in/fan-out device. It is expected that the optimal EVM average performance of LTE-A and WiMAX signals may change considering other MCFs with different length and core pitch. Further investigation will be evaluated in future works considering coupled MCFs with higher fiber lengths and larger bending radius inducing higher crosstalk levels.

\section{REFERENCES}

[1] R.-J. Essiambre, G. Kramer, P. J. Winzer, G. J. Foschini, and B. Goebel, "Capacity limits of optical fiber networks," OSA/IEEE J. Lightw. Technol., vol. 28, no. 4, pp. 662-701, February 2010.

[2] P. J. Winzer, "High-spectral-efficiency optical modulation formats," OSA/IEEE J. Lightw. Technol., vol. 30, no. 24, pp. 3824-3835, December 2012.

[3] D. J. Richardson, et al., "Space-division multiplexing in optical fibres," Nature Photon., vol. 7, pp. 354-362, 2013.

[4] R.G.H. van Uden, R. A. Correa, E.A. López, F.M. Huijskens, C. Xia, G. Li, A. Schülzgen, H. de Waardt, A.M.J. Koonen, and C.M. Okonkwo, "Ultra-high-density spatial division multiplexing with a few-mode multi-core fibre," Nature Photon., vol. 8, pp. 865-870, 2014.

[5] K. Tanaka and A. Agata, "Next-generation Optical Access Networks for C-RAN," presented at the Optical Fiber Communication Conf. Exhibition, Los Angeles, CA, USA, paper Tu2E.1, March 2015.

[6] N. Cvijetic, "Optical network evolution for 5G mobile applications and SDN-based control", in Proc. 16th International Telecomm. Network Strategy and Planning Symposium (Networks 2014), Sept. 2014.

[7] P. Öhlen, B. Skubic, A. Rostami, K. Laraqui, F. Cavaliere, B. Varga, and N. Fonseca, "Flexibility in 5G transport network: the key to meeting the demand for connectivity," Ericsson Technology Review, Oct. 2015.

[8] T. S. Rappaport, "Millimeter Wave Wireless Communications for 5G Cellular: It will work!," presented at the Personal, Indoor, and Mobile Radio Communications Conference, Keynote presentation, 2014.

[9] Ericsson. "5G Energy Performance," Ericsson white paper, April 2015.

[10] U. Kohn, "Fronthaul networks -a key enabler for LTE-Advanced," ADVA Optical Networking technology white paper, Feb. 2014.

[11] M. Zhu, X. Liu, N. Chand, F. Effenberger, and G.-K. Chang, "Highcapacity mobile fronthaul supporting LTE-Advanced carrier aggregation and $8 \times 8$ MIMO," presented at the Optical Fiber Communication Conf. Exhibition, paper M2J.3, 2015.
[12] L. Cheng, X. Liu, N. Chand, F. Effenberger, and G.-K. Chang, "Experimental demonstration of sub-Nyquist sampling for bandwidthand hardware-efficient mobile fronthaul supporting $128 \times 128$ MIMO with $100-\mathrm{MHz}$ OFDM signals," presented at the Optical Fiber Communication Conf. Exhibition, paper W3C.3, 2016.

[13] N. Wang, E. Hossain, and V. K. Bhargava, "Backhauling 5G small cells: A radio resource management perspective," IEEE Wireless Communications, vol. 22, no. 5, pp. 41-49, 2015.

[14] M. Morant, A. Macho and R. Llorente, "Optical fronthaul of LTEAdvanced MIMO by spatial multiplexing in multicore fiber," presented at the Optical Fiber Communication Conf. Exhibition, Los Angeles, CA, USA, paper W1F.6, 2015.

[15] M. Morant, A. Macho, and R. Llorente, "On the suitability of multicore fiber for LTE-advanced MIMO optical fronthaul systems," OSA/IEEE J. Lightw. Technol., vol. 34, no 2, pp. 1-7, 2016.

[16] A. Karadimitrakis, A. L. Moustakas, and P. Vivo, "Outage capacity for the optical MIMO channel," IEEE Transactions on Information Theory, vol. 60, no. 7, pp. 4370-4382, 2014.

[17] A. Sano, H. Takara, Y. Moyamoto, "Large capacity transmission systems using multi-core fibers," presented at the OptoElectronics and Communication Conf., Melbourne, Australia, pp. 704-705, July 2014.

[18] T. Mizuno, H. Takara, A. Sano, and Y. Miyamoto, "Dense space division multiplexed transmission over multi-core and multi-mode fiber," presented at the Optical Fiber Communication Conf. Exhibition, Los Angeles, CA, USA, paper Th1D.2, March 2015.

[19] M. Koshiba, K. Saitoh, K. Takenaga, and S. Matsuo, "Multi-core fiber design and analysis: coupled-mode theory and coupled-power theory," OSA Opt. Exp., vol. 19, no. 26, pp. B102-B111, November 2011.

[20] A. Macho, M. Morant, and R. Llorente, "Experimental evaluation of nonlinear crosstalk in multi-core fiber," OSA Opt. Exp., vol. 23, no. 14, pp. $18712-18720$, July 2015.

[21] A. Macho, M. Morant, and R. Llorente, "Unified model of linear and nonlinear crosstalk in multi-core fiber," OSA/IEEE J. Lightw. Technol. (accepted to be published), 2016.

[22] J. T. J. Penttinen, The LTE-Advanced Deployment Handbook, USA: John Wiley \& Sons, 2015, ch. 7.

[23] X. Ligeret, "Mobile terminating network failure forwarding for LTE circuit-switched fallback", US 20150271711 A1, US 14/224,044 patent, Sept. 2015

[24] A. G. Sarigiannidis, M. Iloridou, P. Nicopolitidis, G. Papadimitriou, F.-N. Pavlidou, P. G. Sarigiannidis, M. D. Louta, and V. Vitsas, "Architectures and bandwidth allocation schemes for hybrid wirelessoptical networks", IEEE Communications Surveys\&Tutorials, vol. 17, no. 1, pp. 427-468, 2015.

[25] M. Morant, J. Prat and R. Llorente, "Radio-Over-fiber optical polarization-multiplexed networks for 3GPP wireless carrier-aggregated MIMO provision," OSA/IEEE J. Lightw. Technol., vol. 32, no 20, pp. $3721-3727,2014$

Andrés Macho (S'14) received the Telecommunication Engineering degree (M.Sc.) from the ETSIT-Universidad Politécnica de Madrid in 2013. He worked in 2014 at Telefonica R\&D Corporation in the Core Network Evolution group and he collaborated in some R\&D European projects such as FP7-STRAUSS, FP7-DISCUS and H2020-INSPACE.

Currently, he is working towards the Ph.D. degree at Valencia Nanophotonics Technology Center at the Universitat Politècnica de València. His professional interest includes high-capacity wavelength-division multiplexed networks (WDM), modal analysis of multi-core fibers, advanced optical modulation formats and space-division multiplexing optical transmissions. 
Maria Morant (S'07-M'12) received the M.Sc. degree in Telecommunication Engineering in 2008 and obtained the International Ph.D. degree from the Universitat Politècnica de València, Spain, in 2012. Since 2006, she has investigated optical techniques for the transmission of OFDM-based signals in access networks at Valencia Nanophotonics Technology Center (NTC).

She is currently a post-doc researcher at NTC leading the research project OESED "Optically enhanced spectral coherent detection" dealing with advanced optical sensing. She has participated in European projects dealing with optical communications and optical sensing such as FP7-ICT-FIVER, FP7-IST-UCELLS and FP6-IST-UROOF and in national projects like ULTRADEF, VISICONEC and HIDRASENSE. She has contributed with more than 75 papers to international journals and hot-topic conferences on optical communications. Her current research areas of interest include advanced modulations and optical sensing techniques.

Roberto Llorente (S'99-S'06), M.Sc. degree in Telecommunication Engineering from the Universitat Politècnica de València (UPV), Spain, received the European $\mathrm{Ph} . \mathrm{D}$. degree from the same University in 2006. Since then, he has been in research positions within the university, and in 2002 he joined the Valencia Nanophotonics Technology Center (NTC) as research associate.

Currently, he is professor in the UPV teaching Optical Communications-related subjects. He is also Head of the Optical Systems and Networks Unit in the NTC. He has been leading NTC activities in the European projects FP5-ISTTOPRATE and FP6-ISTUROOF between others. He has proposed and coordinated FP7-IST-UCELLS and FP7-ICTFIVER projects in the field of radio-over-fibre technology and next-generation FTTH networks. Currently, he is leading NTC activities in national and international research projects dealing with optical signal processing applied to optical networks and next-generation test $\&$ measurement.

$\mathrm{He}$ has authored or co-authored more than 150 papers in leading international journals and conferences and has authored three patents. His current research interest includes optical and electro-optical processing applied to optical core and metro networks, hybrid wireless-optical access networks, optical sensing, metrology, and medical imaging systems. 